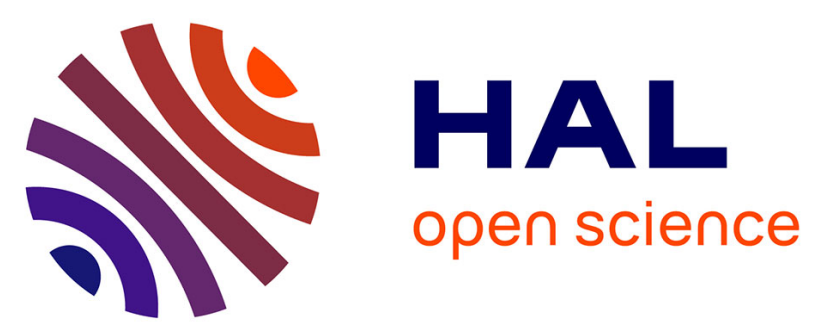

\title{
Analyse médico-économique d'un service de neurochirurgie en centre hospitalier et universitaire
}

Jean-Jacques Lemaire, Cyril Delom, Aurélien Coste, Toufic Khalil, Jean

Christophe Jourdy, Bénédicte Pontier, Jean J Gabrillargues, Denis Sinardet, Abdelkader Chabane, Viorel Achim, et al.

\section{To cite this version:}

Jean-Jacques Lemaire, Cyril Delom, Aurélien Coste, Toufic Khalil, Jean Christophe Jourdy, et al.. Analyse médico-économique d'un service de neurochirurgie en centre hospitalier et universitaire. Réunion de la Société de Neurochirurgie de Langue Française, Société de Neurochirurgie de Langue Française (SNCLF), Dec 2014, Montrouge, France. pp.324-325, 10.1016/j.neuchi.2014.10.012 . hal01870893

\section{HAL Id: hal-01870893 https://hal.science/hal-01870893}

Submitted on 8 Nov 2018

HAL is a multi-disciplinary open access archive for the deposit and dissemination of scientific research documents, whether they are published or not. The documents may come from teaching and research institutions in France or abroad, or from public or private research centers.
L'archive ouverte pluridisciplinaire HAL, est destinée au dépôt et à la diffusion de documents scientifiques de niveau recherche, publiés ou non, émanant des établissements d'enseignement et de recherche français ou étrangers, des laboratoires publics ou privés. 


\section{Analyse médico-économique d'un service de neurochirurgie en centre hospitalier et universitaire}

Lemaire J.-J., Delom C., Coste A., Khalil T., Jourdy C., Pontier B., Gabrillargues J., Sinardet D., Chabane A., Achim V., Sakka L., Coste J., Chazal J., Salagnac A., Coll G., Irthum B.

CHU de Clermont-Ferrand, Clermont-Ferrand, France

Introduction. Les contraintes économiques et sociétales imposent de prendre en compte la dimension économique et la performance médicale. Nous avons ainsi réalisé un travail d'auto-évaluation qu'il nous a semblé intéressant de partager avec la communauté neurochirurgicale.

Matériel/méthode. L'activité de soin et de recherche et sa valorisation ont été étudiées de 2009 à 2013. Nous avons utilisé des paramètres institutionnels et d'organismes évaluateurs pour décrire activités et valorisation. II s'agit d'une étude descriptive rétrospective suivant les recommandations de la Circulaire DHOS/O4 no 2007/390 du 29 octobre 2007.

Résultats. Le compte de résultat analytique moyen annuel était de $+1,39$ millions d'Euros pour 63 lits dont le taux d'occupation moyen corrigé était de $92 \%$, avec 6,7 équivalent - temps plein neurochirurgiens (assistants inclus), concernant 2553 patients et 1975 interventions. Le taux de mortalité (hors 3 lits d'unité de soin continue) était de 2,74\%. La durée moyenne de séjour était de 6,82 jours. Annuellement, en moyenne $15,6 \%$ des patients ont été admis en urgence, et 76,9\% sont sortis au domicile. La valorisation moyenne annuelle de la tarification liée à l'acte et de l'activité de publication représentaient respectivement $77 \%$ et $0,6 \%$ des ressources totales. La difficulté de trouver des lits d'aval pour les patients lourds a été à l'origine de 1401 « journées d'attente » en 2012.

Conclusion. L'analyse médico-économique d'un service de neurochirurgie en centre hospitalier et universitaire rend compte précisément des activités de soin, d'enseignement et de recherche, et de leurs valorisations financières.

Déclaration d'intérêts. Les auteurs déclarent ne pas avoir de conflits d'intérêts en relation avec cet article. 